\title{
Superelastic Deformation Behaviors Based on Phase Transformation Bands in TiNi Shape Memory Alloy
}

\author{
Elzbieta A. Pieczyska ${ }^{1,2, *}$, Hisaaki Tobushi ${ }^{1}$, Stefan P. Gadaj ${ }^{2}$ and Wojciech K. Nowacki ${ }^{2}$ \\ ${ }^{1}$ Department of Mechanical Engineering, AICHI Institute of Technology, Toyota 470-0392, Japan \\ ${ }^{2}$ Institute of Fundamental Technological Research, Polish Academy of Sciences, Swietokrzyska 21, 00-049 Warsaw, Poland
}

\begin{abstract}
Properties and characteristics of superelastic deformation behavior based on Lüders-Like phase transformation bands in TiNi shape memory alloy (SMA) are presented. Temperature distributions accompanying the stress-induced phase transformations in the SMA are found using the infrared technique and employed for the investigation into nucleation and further development of the bands of martensitic and reverse transformations. Based on the temperature and the relevant mechanical characteristics it is noticed that just after crossing a certain threshold stress, narrow bands of considerably higher temperature, about $8 \mathrm{~K}$, corresponding to the martensitic phase, appear starting from the central part of the specimen and developing towards the both specimen borders. A few such bands parallel to each other occur at higher stresses and move towards the specimen grips, as well as their next generation, developing in almost perpendicular direction. The heterogeneous field of the temperature distribution was observed also during the unloading process, while the reverse transformation occurred, also inhomogeneous and related to the significant temperature decrease. Based on the tests carried out with various strain rates, an influence of the strain rate on the mechanical behavior was presented. Thermomechanical aspects of the martensitic and the reverse transformations were discussed.
\end{abstract}

(Received October 7, 2005; Accepted November 21, 2005; Published March 15, 2006)

Keywords: superelastic deformation, shape memory alloy, phase transformation bands, temperature change, infrared camera

\section{Introduction}

Development of the shape memory alloys (SMAs) towards applications to sensors, actuators, damping elements, robots, solid-state heat engines or other "smart" structures depends on their properties found in various conditions. The phenomenon of shape memory in SMAs is associated with a reversible phase transformation. Mechanism of the phase transformation, its nucleation, development as well as the question how far the process is homogeneous, are still a subject of current studies.

Interesting results on the nucleation and propagation of the stress-induced phase transformation in TiNi SMA were presented by Shaw and Kyriakides ${ }^{1)}$ and by Shaw. ${ }^{2)}$ It was found that the superelastic deformation of the SMA is rather inhomogeneous process; it is started from the grip area and developed to the central part of the specimen. In recent works on superelastic deformation behavior in TiNi shape memory alloy of Sun ${ }^{3)}$ and Pieczyska et al., ${ }^{4)}$ it have been noticed that after the elastic deformation followed by rather uniform stage of very initial phase transformation, the main transformation occur in inhomogeneous way, via subsequent inclined narrow bands of the new phase, characterized by different temperature from the rest part of the specimen. Depending on the current equilibrium conditions, it can start from the central part of the specimen or from the grip area. In the results presented by Pieczyska et al.,5,6) after nucleation of the inhomogeneous phase transformation process, the subsequent bands of the new phase appeared in two almost perpendicular directions, and developed towards the specimen grip area. Attention of the present paper is focused on the superelastic deformation behavior of TiNi shape memory alloy subjected to strain-controlled tests with various strain rates, based on the phase transformation bands monitored by thermovision camera.

*Corresponding author, E-mail: epiecz@aitech.ac.jp

\section{Experimental Procedure}

The tests of TiNi SMA of the constitution: Ti-55.3 wt $\%$ Ni were performed on a belt type specimens of dimensions $160 \mathrm{~mm} \times 10 \mathrm{~mm} \times 0.4 \mathrm{~mm}$, cut from a strip of the same cross section. The material subjected to testing is characterized by the austenite finish temperature $A_{\mathrm{f}}$ equal to $283 \mathrm{~K}$. Since the $A_{\mathrm{f}}$ temperature is so low, the TiNi SMA demonstrates a complete loop of pseudoelasticity during the tests carried out at room temperature. Before the testing, the specimen surface was covered with a very thin layer of carbon black powder to make its emissivity higher and homogeneous in order to increase the temperature measurement accuracy.

Scheme and photograph of the experimental set-up are shown in Figs. 1 and 2.

The applied research methodology is based on the experimental scheme as follows. A specimen of the TiNi SMA was loaded by the testing machine in order to obtain the

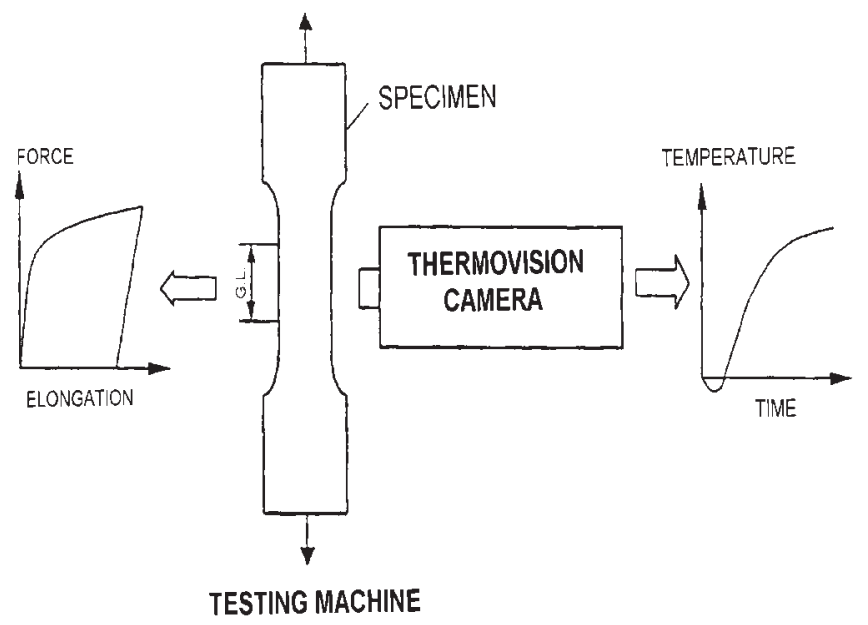

Fig. 1 Scheme of the experimental set-up. 


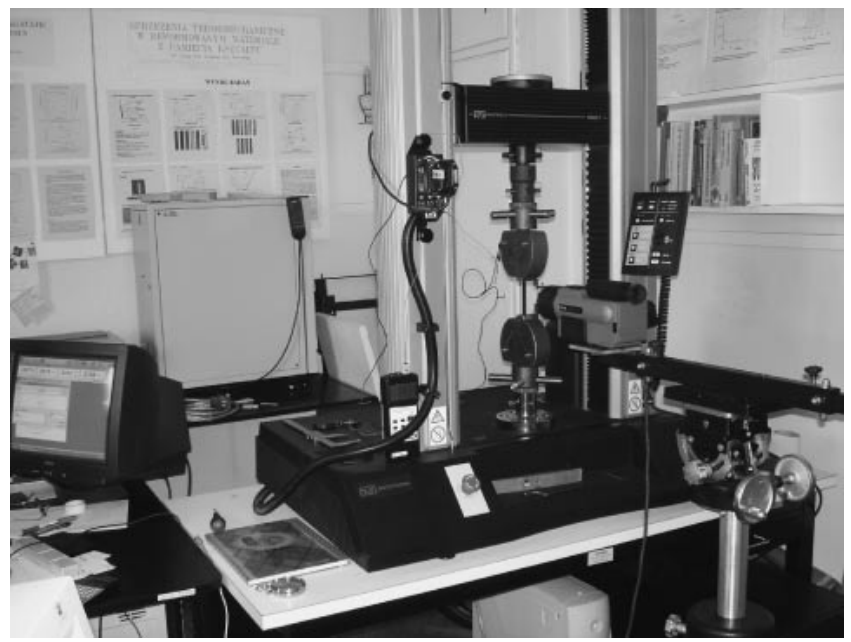

Fig. 2 Photograph of the testing equipment-with a specimen in testing machine and infrared camera.

stress-strain characteristics with a high accuracy. The infrared camera was used in order to find the temperature distributions of the specimen subjected to loading. It registers a distribution of infrared radiation from the specimen surface and creates thermograms with $50 \mathrm{~Hz}$ frequencies. A computer system of the data acquisition and conversion enables to register, store and reconstruct the obtained mechanical and temperature data. In this way, the temperature changes can be presented as a function of various mechanical parameters in any time, as required.

The specimens were subjected to the strain-controlled tension tests in the quasi-static range of deformation, with the strain rates equal to $5 \times 10^{-4} \mathrm{~s}^{-1}, 5 \times 10^{-3} \mathrm{~s}^{-1}, 5 \times 10^{-2} \mathrm{~s}^{-1}$ and $10^{-1} \mathrm{~s}^{-1}$. Three kinds of temperature registration were applied for these investigations:

(1) temperature distribution on the specimen surface,

(2) mean temperature taken from the chosen specimen area,

(3) change in temperature of a chosen point on the specimen surface.

The temperature distribution on the specimen surface immediately reflects the origin and development of the new phases, both martensite and reverse, due to the significant temperature variations between the parent and the new phase.

The average temperature, used in the analysis, was calculated over an area of $8 \mathrm{~mm} \times 60 \mathrm{~mm}$ dimension located in the central part of the specimen. The accuracy of the temperature measurement is $0.1 \mathrm{~K}$.

The point temperature was taken from this part of the specimen surface where the first band of the higher temperature, related to the new martensite phase, was noticed.

The temperatures and the mechanical characteristics related to them enable analysis of the process of nucleation and further development of both the martensitic and the reverse transformations.

\section{Superelastic Deformation Behaviors}

The obtained mechanical and the temperature characteristics as well as numerous thermograms showing temperature

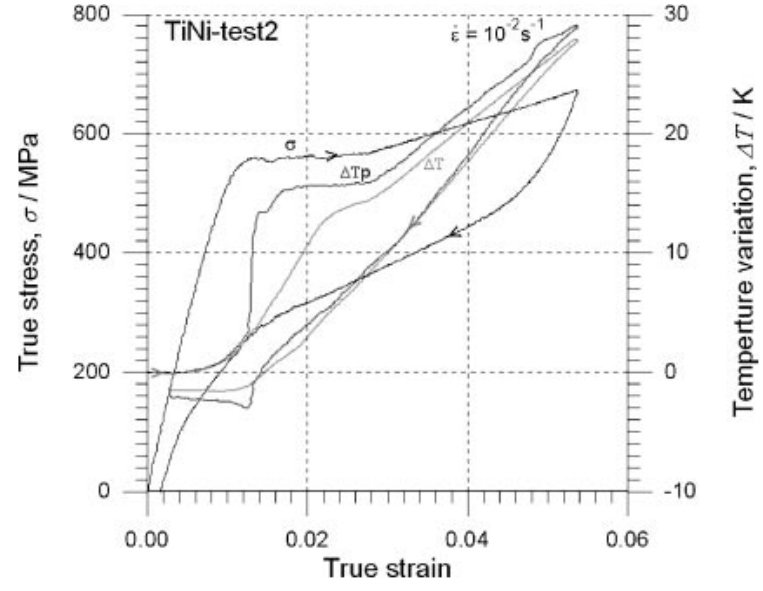

Fig. 3 Stress-strain curve and temperature changes of TiNi SMA: $\Delta T$ denotes average temperature change from the specimen area and $\Delta T_{\mathrm{P}}$ denotes temperature change at the point where the phase transition start was noticed.

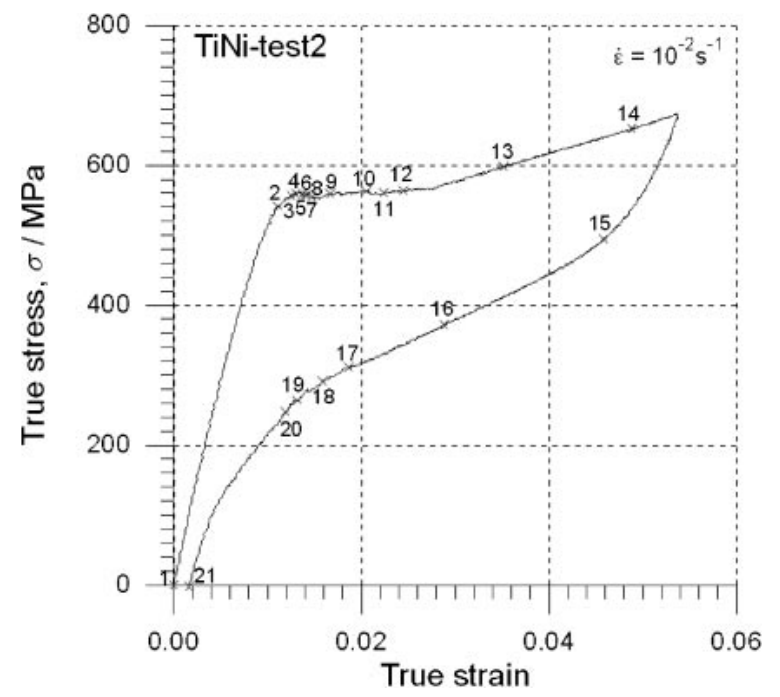

Fig. 4 Stress-strain curve under strain-controlled conditions of TiNi SMA with the strain rate $10^{-2} \mathrm{~s}^{-1}$; marked points correspond to thermograms shown in Figs. 5-10.

distributions on the surface of the specimen are shown in Figs. 3-12. An example of the worked out mechanical and the temperature curves during the complete loop of the phase transformation process is shown in Fig. 3: $\Delta T$ denotes the average temperature calculated for the chosen specimen area, while $\Delta T_{\mathrm{P}}$ denotes the temperature taken from the point where the first signal of significantly higher temperature related to the new phase was noticed.

After the initial, homogeneous stage of deformation, a little overshoot followed by a waving stress plateau related to the inhomogeneous martensitic transformation was observed. Next, the stress-strain curve becomes more smooth and its slope of inclination higher. During unloading, the reverse transformation occurs. It was found that the both phase transformations were accompanied by significant temperature changes; the martensitic transformation was accompanied by the temperature increase up to $29 \mathrm{~K}$ for this strain 
1

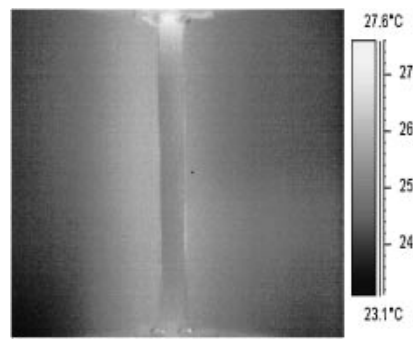

2

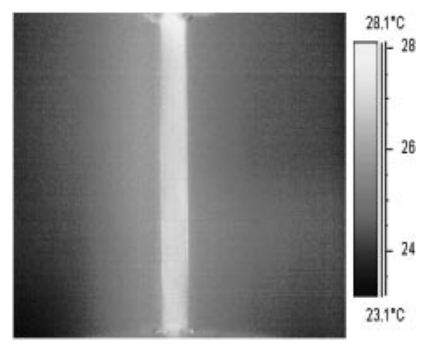

3

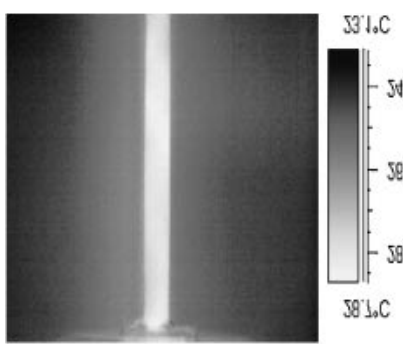

Fig. 5 Temperature distribution of TiNi SMA subjected to stress-induced phase transformation during the initial part of loading. Numbers 1, 2, 3 correspond to the proper points of the stress-strain curve shown in Fig. 4.

4
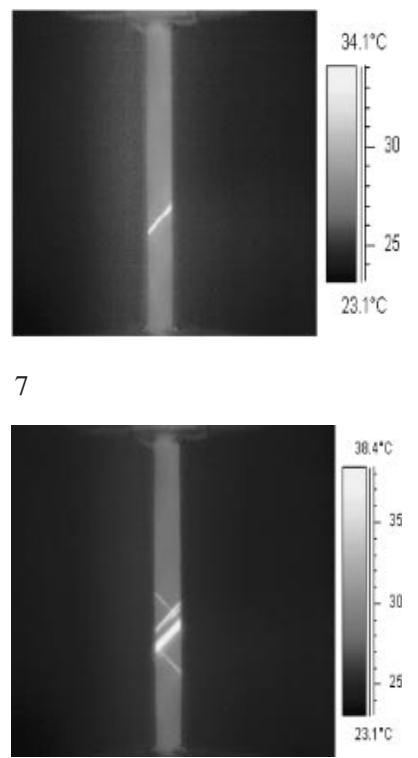

5

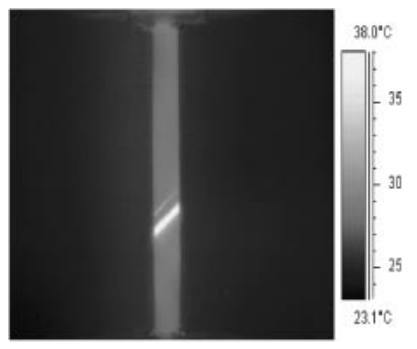

8

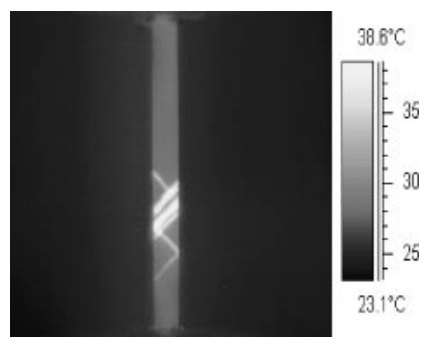

6

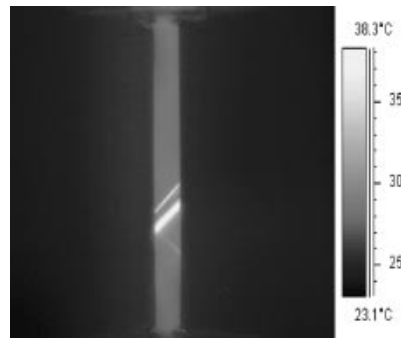

9

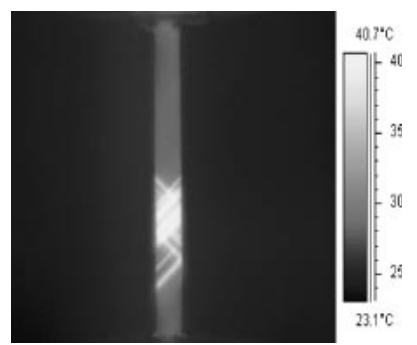

Fig. 6 Temperature distribution of TiNi SMA subjected to stress-induced martensitic transformation. The numbers above the thermograms correspond to the proper points of the stress-strain curve shown in Fig. 4.

rate, while the reverse transformation was accompanied by the significant temperature decrease.

Taking advantages from the infrared camera which monitored the specimen surface and registered the temperature distribution, the phenomena related to the martensitic and the reverse transformations were analyzed.

\subsection{Martensitic transformation}

Before the tension start the specimen temperature was equal to the ambient temperature of $296 \mathrm{~K}$ : Fig. 3. In initial range of the deformation, the temperature distribution was uniform, which was pointed out on the homogeneity of the stress and the strain state along the specimen: Fig. 5; picture 1 . When the stress increases up to its local maximum (see Figs. 3 and 4) the specimen temperature increased, the thermal image, however, remained almost quite uniform indicating homogeneous nature of the deformation process: Fig. 5; picture 2. Next, the temperature slightly increased in the central area of the sample: Fig. 5; picture 3, which was followed by a sudden occurrence of the first inclined band of significantly higher temperature: Fig. 6; picture 4. This was the onset of the inhomogeneous martensitic transformation.

A sudden increase in temperature, related to this band, was equal to about $8 \mathrm{~K}$. After a while, a few such bands parallel to each other occurred moving towards the specimen borders: Fig. 6; pictures 5, 6, as well as their next generation, developing in almost perpendicular direction: Fig. 6; pictures $7-9$. The narrow bands, characterised by significantly higher temperature, immediately evolved into wider ones. The highest temperature, up to $30 \mathrm{~K}$, was registered in the intersection of the bands.

When the strain increased, a still higher number of such bands of the new material phase, characterized by a significantly higher temperature, were registered. Nevertheless, it was observed for all the tests, that the phase transition started in the central area of the sample and developed in both directions towards the grips (Figs. 5, 6 and 7). After this stage, just when the bands of the new phase reached the specimen grips (Fig. 7; picture 12), the angle of inclination of the stress-strain curve started to be slightly higher and the curve was smoother (Figs. 3 and 4). The highest temperature, probably related to the still developing phase transformation, 
10

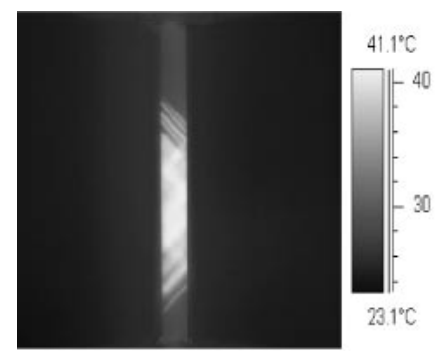

11

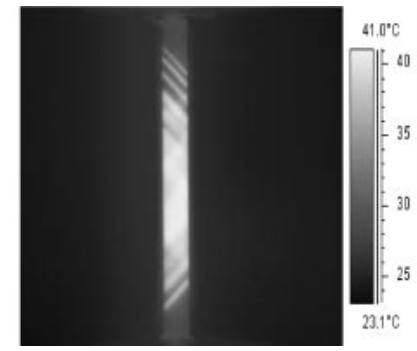

12

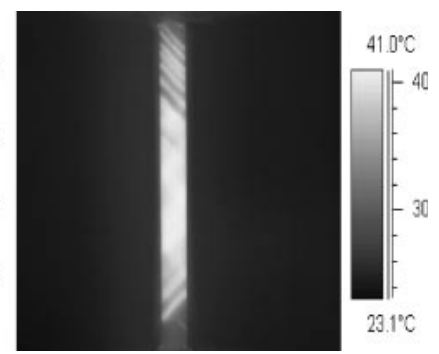

Fig. 7 Temperature distribution of TiNi SMA subjected to stress-induced phase transformation during martensitic transition. The numbers above the thermograms correspond to the proper points of the stress-strain curve shown in Fig. 4.

13

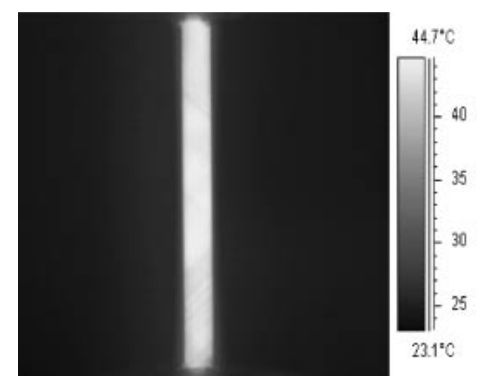

14

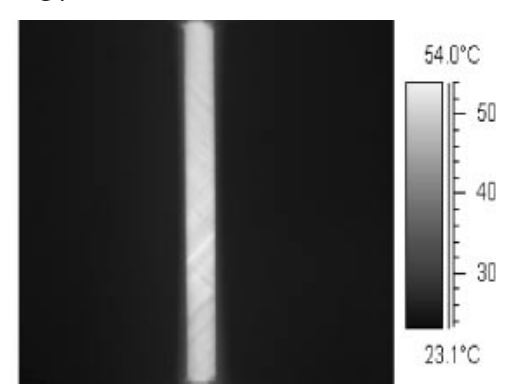

Fig. 8 Temperature distribution of TiNi SMA subjected to stress-induced martensitic transformation. The numbers above the thermograms correspond to the proper points of the stress-strain curve shown in Fig. 4.

was observed and registered in the central area of the specimen.

At higher strains, the regions of higher temperature became less clearly defined, most likely because of the further development of the martensite phase in the whole material volume and the heat flow to the surroundings. When the martensitic transformation was almost completed, the temperature of the specimen surface became more uniform again (Fig. 8; picture 14). Furthermore, during the following tests carried out on the same TiNi SMA material, it turned out that the specimen could be loading further, in wider strain range, without entering the plastic range of the new martensite phase. It means that the recoverable SMA deformation range could be longer.

\subsection{Reverse transformation}

The heterogeneous field of the temperature distribution, related to the transformation bands, was also observed during unloading, when the reverse transition, accompanied by significant temperature decrease, took place (Figs. 3, 9 and $10)$.

When unloading, the temperature drops as the stress decreases. At first, at the elastic unloading stage, a slow temperature drop is observed due to heat exchange with the surroundings. Then, the temperature drops rapidly due to the endothermic reverse transformation. At the latest stage of the unloading, a drop in the mean specimen temperature slows down remaining at almost the same level until the unloading process ends.

At the beginning of the unloading, the temperature distribution was almost uniform-there were not too many differences on the specimen surface: Fig. 9; pictures 15 and 16. Next, the temperature distribution became inhomogeneous. Suddenly, on the all specimen surface the narrow bands of lower temperature in two almost perpendicular directions are observed. The bands are related to the reverse transformation. During the more advanced process of unloading, at the end of reverse transformation, the registered bands of various colors, related to the different temperature, were almost uniformly distributed on the all specimen surface area: Fig. 9; pictures 18, 19 and 20. The lowest temperature was registered in the intersection of the bands, developing in two almost perpendicular directions.

The temperature distributions registered after complete unloading of the specimen were shown in Fig. 10. After $0.98 \mathrm{~s}$ of unloading: Fig. 10; picture 21a, the temperature distribution was still heterogeneous. Even after $3.20 \mathrm{~s}$ of the unloading to null: Fig. 10; picture 21b, the temperature distribution still remained non-uniform, which was probably related to the residual martensite.

The reverse transformation is accompanied by the temperature decrease (Fig. 3). After the unloading to null, when the reverse transformation was almost completed, the temperature of the specimen falls down with the drop up to $5 \mathrm{~K}$, beyond the initial temperature of the specimen.

\section{Influence of the Strain Rate on the SMA Thermome- chanical Superelastic Behavior}

In order to analyze the influence of the strain rate on the TiNi SMA superelastic behavior, the tension tests were performed with various strain rates: $5 \times 10^{-4} \mathrm{~s}^{-1} ; 5 \times$ 
15

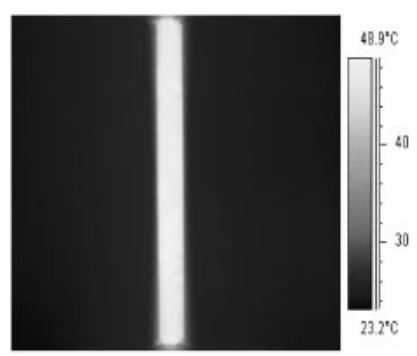

18

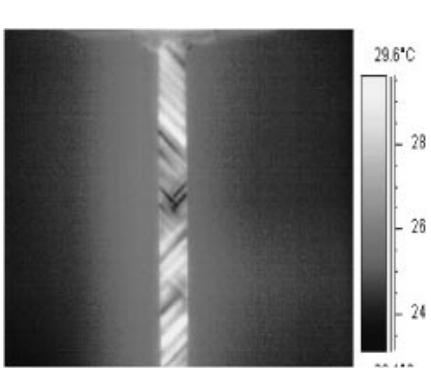

16

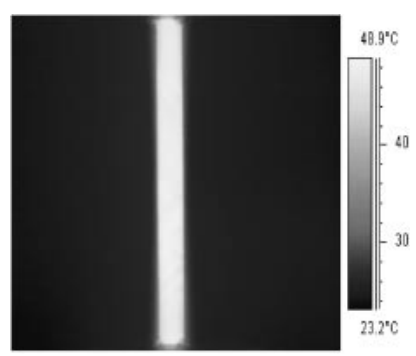

19
17

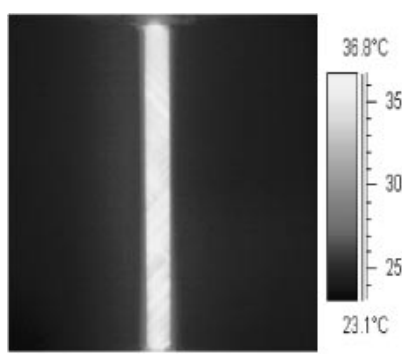

20
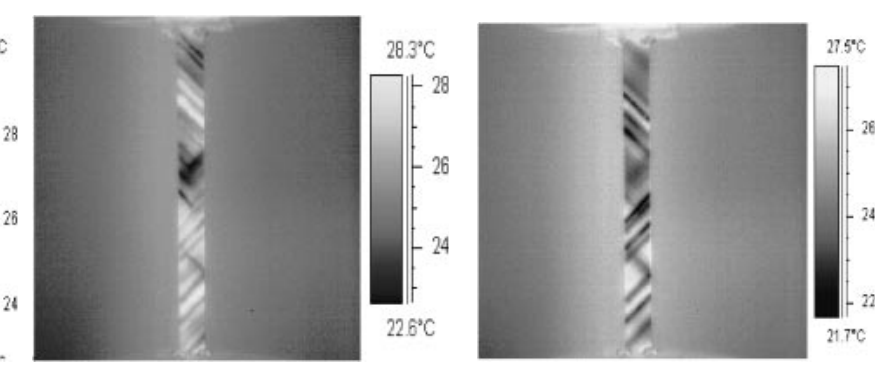

Fig. 9 Temperature distribution of TiNi SMA subjected to stress induced reverse transformation. The numbers above the thermograms correspond to the proper points of the stress-strain curve shown in Fig. 4.

$21 \mathrm{a}$

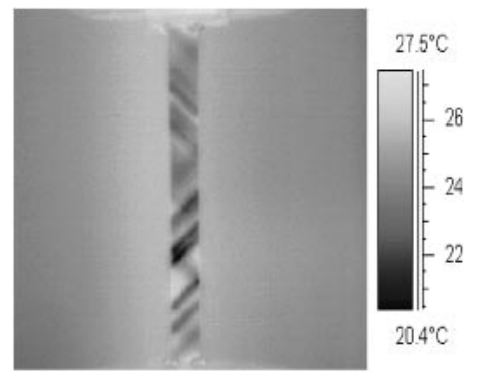

$21 b$

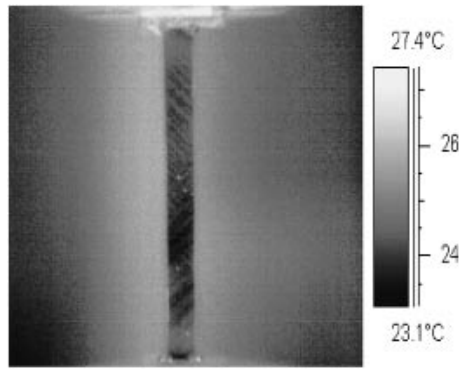

Fig. 10 Temperature distribution of TiNi SMA subjected to stress induced phase transformation after the complete unloading: the numbers above the thermograms correspond to the point 21 of the stress-strain curve shown in Fig. $4 ; 21 \mathrm{a}-0.98 \mathrm{~s}$ and $21 \mathrm{~b}-3.20 \mathrm{~s}$ after the specimen unloading.

$10^{-3} \mathrm{~s}^{-1}, 5 \times 10^{-2} \mathrm{~s}^{-1}$ and $10^{-1} \mathrm{~s}^{-1}$. The obtained stressstrain curves are presented in Fig. 11.

For the all strain rates applied, the pseudoelasticity effects were registered. After an homogeneous deformation the martensitic transformation starts. Its initial stage is manifested by waving part of the stress-strain curves, similar to that observed in Lüders inhomogeneous deformation. Next, the upswing region is observed, related to a more advanced and more uniform phase transformation process.

During the process of unloading, after passing its elastic stage, the reverse transformation initiates. After it is completed, the material almost returns to the parent austenite phase. The specimen also reverts to its initial shape, however the residual strain, probably related to a small amount of residual martensite, depending on the specimen history, usually appears.

During the martensite transformation, stress magnitude increases as the strain rate grows, since the temperature increases while the deformation process develops. This was discussed by Lin et al., ${ }^{7)}$ Tobushi et al. ${ }^{8)}$ Gadaj et al. ${ }^{9)}$ The stress-strain curve developing at a very low strain rate $10^{-4} \mathrm{~s}^{-1}$ is almost flat, like the typical curve illustrating the phenomenon of pseudo-elasticity in SMAs, found for low strain rates: Tanaka et al., ${ }^{10)}$ Tobushi et al., ${ }^{11)}$ Gadaj et al.,${ }^{12)}$ Pieczyska et al. ${ }^{13)}$ For higher strain rates, the stress level is higher and the slopes of inclination of the segments of stressstrain curve, corresponding to the martensitic and the reverse transformations, are significantly steeper; the higher the strain rate, the higher the slope of inclination.

The temperature variations vs. stress found during the tension tests carried out with various strain rates are presented in Fig. 12. After the elastic range of deformation followed by insignificant temperature increase related to the initial, uniform stage of an phase transition, a significant temperature increase, related to the exothermic and inhomogeneous martensitic transformation, is observed. The highest temperature increament is observed at the end of the martensitic transformation and it is changing from 26 to 


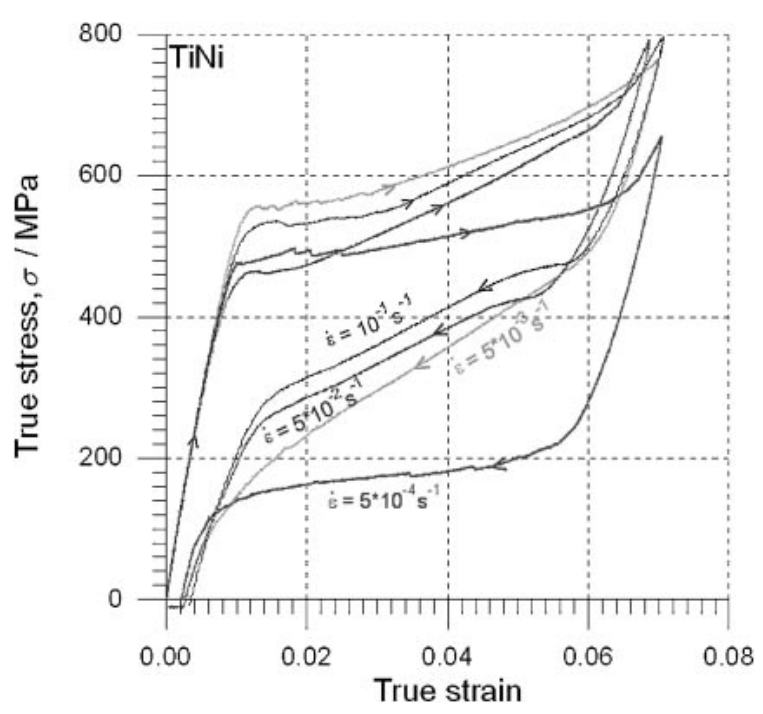

Fig. 11 Stress-strain curves of TiNi SMA subjected to strain-controlled tension tests with strain rates: $5 \times 10^{-4} \mathrm{~s}^{-1} ; 5 \times 10^{-3} \mathrm{~s}^{-1}, 5 \times 10^{-2} \mathrm{~s}^{-1}$, $10^{-1} \mathrm{~s}^{-1}$.

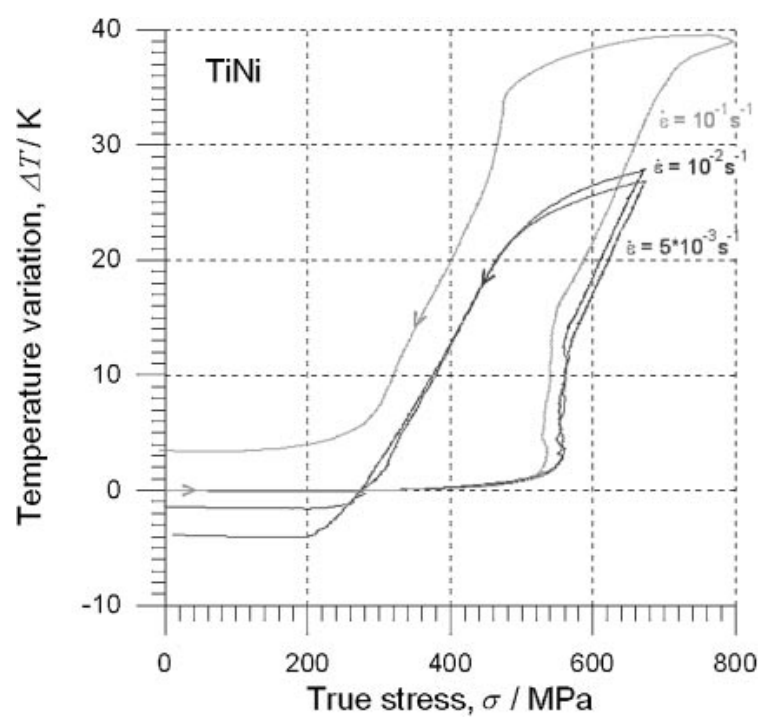

Fig. 12 Temperature changes vs. stress in TiNi SMA subjected to straincontrolled tension test with strain rates $5 \times 10^{-3} \mathrm{~s}^{-1}, 10^{-2} \mathrm{~s}^{-1}$ and $10^{-1} \mathrm{~s}^{-1}$.

$40 \mathrm{~K}$, depending on the strain rate applied; the higher the strain rate the higher the temperature variations. When unloading, the temperature drops as the stress decreases. At first, at the elastic unloading, a slow temperature drop can be observed caused by heat exchange with the surroundings. Then, the temperature drops rapidly due to the endothermic reverse transformation. The segments of curves, related to the phase transformation are almost parallel to each other, irrespective of the strain rate applied. The slope of inclination of the segments, related to the martensitic transformation, is slightly higher than that obtained for the reverse one. After the reverse transformation is completed, the temperature drops below room temperature. The lowest temperature drop of $9 \mathrm{~K}$ was found for the test carried out with the lowest strain rate: $5 \times 10^{-3} \mathrm{~s}^{-1}$. The lower the strain rate, the greater the drop in temperature was registered which is probably caused by the heat transfer and the grip influence, since for the higher strain rate the process is more adiabatic.

\section{Conclusions}

The thermomechanical behavior of TiNi SMA confirmed the exothermic character of the austenite into martensite transformation and the endothermic character of the reverse transformation: the average temperature changes were up to $40 \mathrm{~K}$ for the test carried out with the highest strain rate.

The temperature distributions registered during loading and unloading were not uniform in the ranges of martensite and reverse transformations, which demonstrate that both the transformations occurred in inhomogeneous way, via subsequent bands of new phase, similar to Lüders bands.

The narrow bands of the significantly higher temperature related to the martensitic phase were observed to start from the central part of the specimen and to develop towards the specimen grips; they were characterized by temperature increase of about $8 \mathrm{~K}$; their angle of inclination to the tension direction was about 48 degree. For higher stresses, the bands of new phase were also developed in the perpendicular direction; the highest temperature was registered in the intersection of the bands.

At higher strain, the regions of higher temperature became less clearly defined, most likely because of the further martensite phase development in the whole material volume and the heat flow to the surrounding. When the martensitic transformation was almost completed, the temperature of the specimen surface became almost uniform again.

The bands of reverse transformation observed during the unloading started and developed in whole the material volume, however the process was still inhomogeneous, the bands of new phase were wider in comparison to the martensitic fronts and were characterized by the temperature decrease of about $8 \mathrm{~K}$; their slope of inclination was also about 48 degree.

During the more advanced process of unloading, at the end of reverse transformation, the registered narrow bands of various colors, related to the different temperature, were almost uniformly distributed on the all specimen surface area and the process is more homogeneous.

After complete unloading of the specimen, the temperature distribution was still inhomogeneous which was probably related to the residual martensite, still remained in the specimen.

\section{Acknowledgments}

This research has been carried out with the support of the Polish Ministry of Science under Grants No. 4T08A06024, and of the Japan Society for the Promotion of Sciences under Grants: No. 13650104 of Scientific Research (C), Post-doc ID No. P04774 and Bilateral Program of Joint Research supported by JSPS and PAS, No. 6612.

\section{REFERENCES}

1) J. A. Shaw and S. Kyriakides: Acta Mater. 45 (1997) 683-700. 
2) J. A. Shaw: Plasticity 16 (2000) 541-562.

3) Q. P. Sun: ICTAM04 Abstract and CD-Rom Proceedings, ed. by W. Gutkowski and T. K. Kowalewski, (The IPPT PAN, Warsaw, 2004) 305.

4) E. Pieczyska, S. Gadaj, W. Nowacki and H. Tobushi: QIRT J. 1.1 (2004) 117-128.

5) E. A. Pieczyska, S. P. Gadaj, W. K. Nowacki and H. Tobushi: Bull PAS 3 (2004) 165-171.

6) E. A. Pieczyska, S. P. Gadaj, W. K. Nowacki and H. Tobushi: Proc. 6th Int. Conf. on Intelligent Materials and Systems, ed. by M. Aizawa, (The Intelligent Mater. And Systems Forum, The Soc. For Non-Traditional Technology, Tokyo, 2005) pp. 163-166.

7) P. Lin, H. Tobushi, A. Ikai and K. Tanaka: J. Appl. Biomechanics. 10
(1995) 1-11

8) H. Tobushi, K. Takata, Y. Shimeno, W. K. Nowacki and S. P. Gadaj: Proc. Instn. Mech. Engrs., Vol. 213, Part L, (1999) pp. 93-102.

9) S. P. Gadaj, W. K. Nowacki and H. Tobushi: Arch. Mech. 51 (1999) 649-663.

10) K. Tanaka, S. Kobayashi and Y. Sato: Inter. J. Plasticity 2 (1986) 5972.

11) H. Tobushi, K. Okumara, M. Endo and K. Tanaka: Arch. Mech. 54 (2002) 75-91.

12) S. P. Gadaj, W. K. Nowacki and E. A. Pieczyska: Infrared Physics \& Tech. 43 (2002) 151-155.

13) E. A. Pieczyska, S. P. Gadaj and W. K. Nowacki: Proc. of SPIE's AeroSense 4710 (2002) 479-487. 